\title{
À propos de la société rurale en contexte d'urbanisation
}

\section{Claire Dolan}

Volume 45, numéro 1, été 1991

URI : https://id.erudit.org/iderudit/304949ar

DOI : https://doi.org/10.7202/304949ar

Aller au sommaire du numéro

Éditeur(s)

Institut d'histoire de l'Amérique française

ISSN

0035-2357 (imprimé)

1492-1383 (numérique)

Découvrir la revue

Citer cette note

Dolan, C. (1991). À propos de la société rurale en contexte d'urbanisation. Revue d'histoire de l'Amérique française, 45(1), 101-109.

https://doi.org/10.7202/304949ar d'utilisation que vous pouvez consulter en ligne.

https://apropos.erudit.org/fr/usagers/politique-dutilisation/ 


\section{NOTE CRITIQUE}

\section{À PROPOS DE LA SOCIÉTÉ RURALE EN CONTEXTE D'URBANISATION}

Que de chemin parcouru depuis que le groupe franco-québécois d'histoire rurale s'est attaqué à l'analyse des sociétés rurales de part et d'autre de l'Atlantique, dans une perspective comparative! C'est le constat auquel conduit la lecture des actes de son dernier colloque, tenu en février $1990^{1}$.

Publiés sans délai, ces actes fournissent une série d'instantanés de la recherche poursuivie par les membres du groupe. Non seulement l'histoire, mais aussi la sociologie et l'ethnologie, l'anthropologie et l'histoire du droit y observent, chacune de leur point de vue, les transformations de la société rurale. Des unes et des autres, on tirera des informations, des interprétations, mais de l'ensemble, on dégagera la nécessité de leur mise en relation. La sociologie a fourni la théorie de la reproduction sociale qui donne à l'ouvrage sa principale unité. L'ethnologue, l'anthropologue et l'historien bénéficient des effets d'une longue familiarité qui permet de combiner harmonieusement leurs apports respectifs. Plus récente, la collaboration avec l'histoire du droit a besoin d'être mieux rôdée: question de langage, question d'état d'esprit. Les historiens doivent cesser de considérer le droit comme une énigme obscure, les historiens du droit cesser d'entretenir ce mythe.

L'ouvrage réunit les matériaux: au lecteur de les utiliser pour effectuer les rapprochements entre les régions. Disparue la proclamation des bonnes intentions comparatives qui parsemaient les ouvrages antérieurs! Si l'esprit qui préside aux travaux reste essentiellement comparatif, les termes de la comparaison se sont modifiés. Ce n'est plus deux sociétés rurales, l'une québécoise, l'autre française que l'on confronte mais des dizaines de microsociétés dont les recherches récentes ont révélé les particularités.

1 Gérard Bouchard et Joseph Goy, dir., Famille, économie et société rurale en contexte d'urbanisation (17e -20 siècle), avec la collaboration de J.-P. Wallot, J. Dickinson, J. Roy. Actes du colloque d'histoire comparée Québec-France, tenu à Montréal en février 1990. Chicoutimi et Paris, Centre interuniversitaire SOREP et Écoles des hautes études en sciences sociales, 1990. $388 \mathrm{p}$. 
On ne cherchera, dans les 28 articles que regroupe l'ouvrage, ni synthèse ni généralités sur la société rurale. Chaque texte porte sur un point précis, sur une région précise. Le tout n'est pas toujours de lecture facile et l'on sera peut-être tenté de limiter la consultation aux articles qui touchent ses intérêts particuliers. Au-delà des études très spécialisées qu'il propose, le livre fournit pourtant l'occasion de mesurer les effets que risquent d'avoir sur l'histoire, les progrès de la' recherche.

\section{L'HISTOIRE DANS UN CUL-DE-SAC?}

Alors qu'on savait bien peu sur la société rurale, les interprétations qu'on en faisait étaient péremptoires et globales. À mesure que les travaux se sont multipliés, que la société a été mieux connue, la prudence et les nuances ont remplacé les explications absolues. Les schémas bien ficelés ont révélé leurs failles et il a fallu tout reprendre. Les meilleurs articles de ce colloque remettent en question des certitudes diverses. Sans iconoclasme vain, ces auteurs interrogent. Ils répondent parfois et leurs voix alors s'entremêlent pour réclamer qu'on s'attarde plus aux particularismes, ou pour exiger qu'on s'élève au-dessus d'eux. Quel que soit le parti choisi, le chercheur est en droit de se demander où tout cela le conduit et comment il pourra rendre intelligibles tant de diversités. En effet, le dialogue de sourds qui s'est installé entre les spécialistes et le lecteur moyen n'a pas épargné l'histoire. Alors que le second ne cherche qu'une clé pour comprendre, on reproche aux premiers de se noyer dans la complexité des explications possibles: rien n'est simple, tout est différent, les facteurs sont multiples et leurs effets peu prévisibles. Le spécialiste qui ne peut plus se résoudre à schématiser se contente de lever le drapeau marqué «Danger». Et il continue à chercher, et à multiplier les observations sans savoir s'il arrivera un jour à trouver une cohérence entre elles.

Cet ouvrage témoigne certes du fait que les sociétés rurales sont multiples et diverses. Il montre également la vanité des explications qui ne considèrent qu'un aspect du monde rural, cet aspect fût-il l'économie. Mais il prouve aussi, dans ses meilleurs morceaux, que des conclusions sont possibles et qu'elles doivent être tirées, malgré les diversités qui les masquent souvent.

\section{LA SOCIÉTÉ RURALE EN CONTEXTE D'URBANISATION}

L'objectif du projet dont ce colloque marque la mi-course était de comparer les modes de reproduction de la famille rurale dans le contexte d'une société globale en pleine urbanisation (p. 1). Si la famille fait l'objet des séances les plus cohérentes, c'est en fait la reproduction sociale en contexte d'urbanisation qui court en filigrane de ce livre. Le concept de reproduction sociale lui-même n'est pas discuté même si Martine Segalen s'interroge sur ce qu'il recouvre et se demande quels sont les critères les plus pertinents pour mesurer cette reproduction (p. 165) alors que J.-P. Wallot et Gilles Paquet en résument les principes dans l'introduction de leur texte (p. 251). 
Les communications sont classées dans l'ordre où elles ont été présentées et le titre des séances coiffe les différentes parties de l'ouvrage. De nombreux tableaux statistiques, graphiques et cartes accompagnent les textes. Avec bonheur, on retrouve dans l'ouvrage une certaine unité chronologique qui permet de comparer des périodes comparables: le $\mathrm{XIX}^{\mathrm{e}}$ et le $\mathrm{XX}^{\mathrm{e}}$ siècles sont privilégiés ce qui marginalise toutefois les quelques textes portant sur la période antérieure. Trois ensembles contribuent à façonner l'unité donnée à l'ouvrage par la problématique de la reproduction et révèlent les progrès énormes de la recherche dans ces domaines: ils portent sur la transmission du patrimoine et sur la reproduction familiale, posent le problème des relations entre les ruraux et les urbains, et lient les niveaux de vie à la pénétration de l'économie de marché dans les campagnes.

Les deux premières séances traitent de la transmission des patrimoines et de la reproduction familiale, au Québec et en France. Les deux communautés de chercheurs travaillent différemment: la France bénéficie du nombre de chercheurs et des fruits d'une longue expérience dans ce type de recherche. Deux textes représentent les travaux québécois, ils portent tous deux sur la donation entre vifs. Gérard Bouchard, à la recherche des mécanismes de changement, y voit l'une des nombreuses modalités qu'emprunte la reproduction familiale dans le territoire neuf qu'est le Saguenay. Les donateurs, les donataires, la donation comme mode d'établissement, son impact économique et social, l'effet de la saturation et les tensions sociales qui en découlent sont brièvement présentés, les 18 tableaux qui accompagnent le texte permettant de gagner du temps. Au terme de l'étude, la comparaison avec d'autres régions impose de considérer l'ancienneté et le mode d'exploitation du sol comme des facteurs déterminants dans la pratique de la donation entre vifs.

Renaud Santerre aborde la donation entre vifs d'un point de vue anthropologique. Ses préoccupations gérontologiques le conduisent à considérer celle-ci comme un véritable système de sécurité de vieillesse en milieu rural. C'est cette hypothèse qui fait tout l'intérêt du texte, c'est elle qui en limite également la portée. En effet, toute l'argumentation vise à nous convaincre que la donation constitue un système. La seule périodisation considérée est donc liée à la mise en place de programmes globaux de sécurité de vieillesse. Les années 1950 constituent la césure déterminante, un nouveau système remplaçant alors celui de la donation entre vifs. Du corpus de 3500 actes dont il tire ses réflexions, on ne sait ce que l'auteur pourra tirer de plus. Pourtant le regard différent proposé par l'auteur suscite suffisamment l'intérêt pour appeler une analyse plus fine où les différences régionales (terroir neuf et ancien) seraient mises en évidence. En effet, si le principe fondamental de l' «antique» donation de ferme est lié au fait que «la sécurité économique du vieillard repose sur la valeur accumulée de son travail pendant la vie active» (p. 44), il n'est pas sûr que ce principe soit aussi ancien que l'auteur le prétende et il est bien possible que l'étude de fermes transmises et retransmises, génération après génération, montrerait que l'échange ne se résout pas ici en termes de valeur accumulée du travail. 
La séance sur la transmission des patrimoines et la reproduction familiale en France vaut, à elle seule, la lecture de l'ouvrage. Le texte de Joseph Goy qui ouvre la section est un état de la question qui ne tait ni les réussites, ni les échecs. Jusqu'aux questions restées sans réponse qui montrent, dans ce texte, la vitalité des recherches sur le problème faites en France. Les interprétations simples ne sont plus de mise; si l'on connaît maintenant les systèmes, on ne sait guère comment les sociétés les adoptent, ni vraiment d'où ils viennent. L'impossibilité de trouver des explications miracles amène historiens et ethnologues à chercher d'autres solutions. L'une d'elles est le changement d'échelle. Goy propose quant à lui d'ouvrir la comparaison à l'Europe entière, la multiplication des monographies distribuées de façon anarchique ayant fait son temps.

L'efficacité d'une observation à échelles multiples est par ailleurs largement démontrée par les autres communications de cette séance. Tiphaine Barthélémy constate au-delà de la grande diversité des pratiques bretonnes, des diversités dans le cadre d'une même commune et cherche, à travers 3 parentèles, les raisons de ces différences. Alors que les trois familles qu'elle étudie de génération en génération présentent sensiblement les mêmes idéaux de justice et d'égalité, les moyens qu'elles prennent pour les atteindre sont passablement différents. Cette particularité ne tient ni à des facteurs géographiques ni à des influences culturelles mais d'abord au fait que chacune de ces parentèles effectue un mouvement opposé dans la hiérarchie sociale. Selon des mouvements très lents, la société tire certaines familles vers le haut, d'autres vers le bas et les pratiques successorales et matrimoniales participent de ces mouvements (p. 65). La réduction d'échelle exige qu'on dépasse les explications maintenant récusées liées à la géographie juridique, mais la vision d'une société composée de consanguins formant des blocs montants alors que d'autres forment des blocs descendants me paraît aussi réductrice qu'une explication exclusivement géographique.

L'article d'Olivier Nougarede est comparable du point de vue de l'échelle au précédent. Il montre toutefois que d'une observation minutieuse peuvent naître des conclusions générales stimulantes. Son histoire d'un lignage lozérien, branche après branche, nous apprend comment aux stratégies matrimoniales et patrimoniales se mêlent les tactiques individuelles. Chaque itinéraire illustre un aspect précis de la formation sociale et l'histoire des exclus de l'ostal n'est pas la moins significative. Exclus d'un système où la propriété est la base de la stratification sociale, ils deviennent dans un système qui accorde à l'efficacité du travail une place fondamentale, ceux qui s'adaptent le mieux. La réussite sociale, maintenant, conclut l'auteur «dépend moins de la taille du patrimoine foncier, que de la capacité [...] à tirer le maximum de revenus de son capital productif» (p. 94). La tendance de l'auteur à faire du «traditionnel» un monolithe et à faire naître les oppositions entre logiques traditionnelles et logiques marchandes à la fin du XIX ${ }^{\mathrm{e}}$ et au début du $\mathrm{XX}^{\mathrm{e}}$ siècles suggère de reprendre la problématique pour les périodes antérieures et de tester, avec des méthodes similaires, dans quelle mesure cette opposition n'apparaît pas bien avant. 
Changement d'échelle que propose aussi Georges Augustins qui cherche pourquoi, malgré les tentatives de la société globale d'imposer à la société paysanne son droit et ses modèles culturels, les particularismes locaux continuent à persister. Augustins propose en fait une théorie des transformations et des permanences. S'il existe dans chaque société un principe qui gouverne les pratiques de transmissions dont les formules empruntent à la société globale, l'originalité de ce principe réside dans la manière, dans l'esprit de l'assemblage. Ce qui appartient en propre à chaque société, c'est le principe de légitimité qui préside à l'agencement des idées et des pratiques. Les pratiques peuvent changer, le principe de légitimité, lui, dure. L'auteur donne deux exemples: les Pyrénées et la Bretagne au XIX ${ }^{e}$ et au $\mathrm{XX}^{\mathrm{e}}$ siècles et montre comment le système s'effrite quand le principe de légitimité sur lequel il reposait se disloque. L'analyse est intéressante en ce qu'elle a le mérite de tabler sur les différences pour construire un modèle. Mais son application est difficile, compte tenu du fait que le principe de légitimité des pratiques d'une société doit être d'abord mis au jour. En ce sens, l'article de Georges Augustins montre bien que de telles interprétations exigent que des recherches minutieuses et nombreuses aient d'abord été menées à terme.

Les recherches sur la transmission du patrimoine en France sont très avancées. Elles s'articulent autour d'une réflexion dont les résultats sont maintenant mesurables. Entre les textes de cette partie plus qu'à l'intérieur de chacun d'eux, les différences d'échelles sont très sensibles et volontaires. Elles imposent la prudence, suscitent les nuances mais ne conduisent pas au cul-de-sac que l'on pourrait craindre. En ce, elles indiquent une voie prometteuse.

Les textes que l'on a rangés sous le titre «Exode rural, urbanisation, nouvelles formes sociales» mettent en évidence le fait qu'en contexte d'urbanisation, les relations entre la ville et la campagne se transforment. L'urbanisation, c'est à la fois la ville qui reçoit les ruraux et la campagne qui devient ville. Marc Saint-Hilaire se place du point de vue des ruraux qui vont vers la ville. Les ruraux du Saguenay qui veulent partir se trouvent face à un choix: aller vers la ville ou vers le front pionnier. La décision sera fonction de l'importance du réseau de parenté, du nombre de garçons, de la situation économique de la famille au moment du départ. Une fois en ville, la prolétarisation massive attend ces ruraux, la région perdant ici son originalité. Ce ne sont pas ces prolétaires qui intéressent Pierre Lanthier et Normand Brouillette mais bien la petite bourgeoisie d'origine rurale de Shawinigan. Leur texte insiste sur le fait que les antécédents ruraux de cette bourgeoisie contribuèrent à l'ascension sociale des individus. L'essentiel de leur argumentation repose toutefois sur des clichés agaçants. La solidarité n'est pas, comme le prétendent les auteurs, un trait culturel propre au monde rural. Entre la ville et la campagne, l'on sait bien qu'il n'y a pas opposition. Rolande Bonnain cherche quant à elle comment les modèles de transmission du patrimoine qu'on retrouve à la campagne sont transposés à la ville. Le groupe de commerçants de bestiaux qu'elle étudie se prête particulièrement bien à l'analyse. Entre la ville et la campagne, il transpose dans le cadre 
urbain des modèles successoraux qui caractérisent le milieu rural et en respecte le principe inégalitaire, à la fois dans la transmission de la profession et dans la transmission du patrimoine. Et pourtant, à la ville, le métier exige qu'on adapte à ses besoins le partage des rôles entre époux et suscite de nouveaux types de régimes matrimoniaux.

La perspective de Martine Segalen est en quelque sorte opposée. Ce n'est plus les ruraux qui vont en ville, mais la ville qui atteint les zones jadis rurales. Les agriculteurs, les vignerons et les charcutiers de Nanterre qui forment le fonds autochtone de ce bourg rural «nourrisseur» de Paris, perdent petit à petit leur poids social, sous la pression démographique qu'exerce le développement urbain. Leur dense réseau de parenté s'oppose à la dispersion sociale des ménages des nouveaux arrivants et finit par les marginaliser. Alors que la ville s'impose, les cultivateurs n'auront bientôt plus qu'à quitter Nanterre. Martine Segalen conclut son analyse en posant les vraies questions: en contexte d'urbanisation, le cadre monographique est-il adéquat pour une observation de la reproduction sociale? Au concept de reproduction sociale ne doit-on pas relier la dynamique qui, si elle s'inscrit dans le temps, fait aussi éclater les espaces? Quels sont ceux qui réussissent leur reproduction sociale? Ceux qui s'incrustent dans leur espace d'origine malgré les transformations qui changent fondamentalement la nature de ce milieu ou ceux qui partent se «reproduire» ailleurs (p. 172)? Autant de questions qui montrent la complexité du problème tout autant que les limites d'une observation aux cadres trop réduits.

Bien qu'il inscrive sa recherche dans la perspective de la modernisation de l'agriculture plutôt que dans celle de la reproduction, Jean Roy fournit, dans son étude de l'enseignement agricole au Québec (p. 307), l'occasion de poursuivre l'interrogation de Martine Segalen. On sait la place fondamentale que tient l'enseignement parmi les instruments de la reproduction. En comparant les clientèles et les objectifs de l'enseignement intermédiaire agricole aux cours à domicile publiés par l'UCC, l'auteur amorce une recherche que l'on voudrait voir s'étendre aux programmes, aux enseignants, aux matières enseignées. Posée en d'autres termes, la question de Martine Segalen rejaillit ici: quelle reproduction les écoles d'agriculture favorisent-elles?

En relation avec le contexte d'urbanisation, le monde rural est un monde perméable. Jusqu'où va cette perméabilité quand on l'observe du point de vue des structures économiques? C'est ce que les interventions regroupées sous le titre "Inégalités, niveaux de vie, groupes sociaux», tentent de cerner. Les trois premières communications, à caractère essentiellement méthodologique, sont à lire d'un trait. Christian Dessureault, Micheline Baulant et John Dickinson cherchent les meilleurs moyens de comparer les niveaux de vie français et québécois à partir de la présence de certains objets dans les inventaires après décès et discutent les catégories d'objets retenus en fonction des spécificités de leur société. Si John Dickinson s'acharne à vouloir mettre au point une grille d'analyse utilisable partout, C. Dessureault et M. Baulant partent d'une base commune qu'ils adaptent et raffinent par la suite chacun de leur côté. Au-delà de la méthode, les résultats auxquels ils parviennent sont assez révélateurs: Dessureault constate que le niveau de vie des paysans 
croît de 1800 à 1840 mais que le genre de vie est d'abord axé sur le nécessaire, mettant en évidence les inégalités considérables entre les journaliers, les cultivateurs et les notables. La distinction sociale de ces derniers se manifeste en effet essentiellement dans la part qu'ils accordent au luxe et à ce que Dessureault appelle la "civilisation». L'analyse est fine et ne confond pas la valeur pécuniaire des objets trouvés avec leur valeur symbolique. De son côté, Micheline Baulant peut d'ores et déjà indiquer qu'entre 1750 et 1780 le niveau de vie de ses paysans de Meaux a augmenté de 7,8\%, les notables étant les grands profiteurs de la hausse. Des progrès de la consommation qu'elle constate, elle conclut à la pénétration du marché dans les campagnes briardes. Dickinson le souligne dans son texte, l'indice de niveau de vie fourni par les inventaires mesure des structures matérielles plus que des conjonctures, mais l'amélioration de cet univers matériel témoigne d'une plus forte intégration au marché des populations rurales. Au cœur de ce marché, les marchands ruraux qu'étudie Claude Pronovost à partir des stocks de leur magasin, de leurs créances et de leurs dettes sont un groupe peu homogène. Débiteurs et créanciers à la fois, ils sont touchés par la conjoncture. L'on s'en doutait bien un peu, ainsi va le commerce!

L'article de Gilles Paquet et J.-P. Wallot dont le titre «Crédit et endettement en milieu rural bas-canadien» ne suggère pas le véritable intérêt, sert de conclusion à cet ensemble. Les auteurs y brossent un tableau des débats historiographiques sur l'économie du XIX ${ }^{\mathrm{e}}$ siècle bas-canadien fort éclairant pour les non-canadianistes ou pour les néophytes. Bien sûr, ils y défendent leur propre position, le choix de leurs sources et donnent les résultats essentiels auxquels ils sont arrivés jusqu'à maintenant, mais la perspective globale dans laquelle ils insèrent leur réflexion est fort intéressante. Au terme de cette partie, le lecteur n'a plus de doute, l'économie de marché a bel et bien pénétré les campagnes du Bas-Canada, dès le début du $\mathrm{XIX}^{\mathrm{e}}$ siècle, accompagnée par la constitution parallèle d'un réseau de crédit qui éclaire cette pénétration. On peut maintenant passer à l'étape suivante et chercher quelles en furent les conséquences.

Les autres communications du colloque s'inscrivent moins bien dans la relative unité des textes sur lesquels je me suis attardée. Corinne Beutler recherche les manifestations de la modernisation de la société rurale dans les mentions de nouveaux modèles de charrues qu'elle relève dans les inventaires de la région de Montréal. Lise St-Georges présente une recherche en cours sur Pointe-aux-Trembles et décrit les mutations qu'elle décèle à partir d'une utilisation des recensements et des inventaires. J. I. Little compare les cycles du travail saisonnier des Canadiens français et des Écossais du canton de Winslow. Il conclut, sans toutefois développer ses arguments que les stratégies différentes développées par les deux communautés ont été essentiellement influencées par des données culturelles. Le danger est grand ici de confondre culture et stéréotype mais le texte présenté ici n'est en fait qu'un résumé d'une démonstration probablement beaucoup mieux étayée ailleurs. L'article de J.-P. Desaive sur la forêt, le seigneur et le paysan en BasseBourgogne est un peu perdu dans cet ensemble disparate. Son principal intérêt est de montrer comment fonctionne la société globale qui, pour gérer 
et exploiter la forêt, à partir de 1670 , utilisera la confrontation entre le roi, les seigneurs, les paysans et les marchands. Les façons de détourner la loi, de condamner ou de ne pas sévir montrent comment les besoins et l'utilisation lucrative de la forêt s'accordent pour finalement faire fondre cette dernière. La confrontation entre les Couillard et leurs censitaires à propos des moulins, au XVIII ${ }^{e}$ siècle, s'inscrit dans un contexte bien différent que Thomas Wien caractérise par l'absence de concurrence de l'État face aux privilégiés des campagnes.

L'ouvrage se termine par trois articles qui s'intègrent assez mal au reste. Les «Filles du Roi» au XVII ${ }^{e}$ siècle d'Yvan Landry, la présence étrangère dans les communautés rurales de la Côte-du-Sud au XVIII ${ }^{e}$ siècle d'Alain Laberge et l'occupation de l'espace dans deux régions de la vallée laurentienne au XVIII ${ }^{\mathrm{e}}$ siècle de Lina Gouger et Geneviève Postolec. Si le dernier article est essentiellement descriptif, celui de Laberge remet en question bien des vieilles certitudes et tout autant de clichés: le monde rural du XVIII ${ }^{e}$ siècle n'était ni homogène, ni fermé.

\section{DE NOUVEAUX ALLIÉS? L'HISTOIRE ET LE DROIT}

Au-delà des informations qu'il recèle, des suggestions méthodologiques qu'il propose, l'ouvrage a l'intérêt de mettre à contribution l'histoire du droit, pour l'instant limitée au contexte français. Le droit n'est pas plus immobile que ne l'est la société. C'est ce qu'ont compris les organisateurs de ce colloque en lui ouvrant leurs portes. Les quatre articles qui font du droit le cœur de leurs propos révèlent toutefois que les ponts jetés entre l'histoire et l'histoire du droit sont de diverses natures. Michelle Salitot travaille sur l'esprit du droit. Elle cherche à montrer que les rapports familiaux du XIX ${ }^{e}$ siècle ont leur origine dans le droit médiéval modifié au XVI ${ }^{\mathrm{e}}$ siècle et qu'ils reposent sur la permanence de rapports de domination et de soumission transférés d'un niveau inter-individuel aux secteurs de production dans le mode de production capitaliste. À Nussey, au XIX ${ }^{\mathrm{e}}$ siècle, le système familial semble un compromis entre des rapports familiaux basés sur le principe d'autorité et un modèle reposant sur l'association. L'originalité du cas est discutable et l'utilisation du droit hors de tout contexte l'est plus encore.

Blandine Vue et Gaspard Beaur insistent sur la multiplicité des recours que le droit permet et sonnent l'alarme quant à la valeur de certaines sources. L'article essentiellement descriptif que présente Blandine Vue explique le fonctionnement de l'indivision dans la région de Langres du XIII ${ }^{\mathrm{e}}$ au $\mathrm{XX}^{\mathrm{e}}$ siècle. Entre les informations du cadastre, celles des actes notariés et celles de la pratique quotidienne, bien des écarts sont possibles et Blandine Vue nous en donne ici un exemple parlant. Quant à l'article de Gérard Beaur, il replace le marché foncier dans un ensemble de procédures où les mouvements de propriétés impliquent diverses formes de transmission. À tous ceux qui croyaient pouvoir étudier le marché foncier à partir des seuls actes qui y font expressément référence, l'auteur assène une leçon de prudence.

Louis Lorvellec traite le droit rural pour lui-même, comme un objet historique et non plus seulement comme un instrument d'analyse. Le droit 
s'inscrit dans des réalités très concrètes, indépendamment des législations. Ainsi montre-t-il que le droit rural ne tentera pas de freiner l'urbanisation, mais essaiera plutôt d'en étendre les avantages et les inconvénients à la population rurale dans son ensemble.

Malgré la présence de ces textes dans l'ouvrage, on ne peut pas dire que l'alliance entre l'histoire du droit et l'histoire soit définitivement scellée. Les uns et les autres ont d'abord à trouver dans cette collaboration un intérêt réciproque, ils pourront ensuite élaborer un langage qui leur permettra de parler la même langue, ce qui est loin d'être le cas maintenant.

\section{CHANGEMENT OU PERMANENCE?}

Remettre en question certitudes et clichés, voilà bien ce que fait ce livre. Sans faire le point sur la recherche en histoire rurale de part et d'autre de l'Atlantique, il donne, par petites touches, des couleurs au tableau et montre que les certitudes à reviser ne sont pas les mêmes d'une historiographie à l'autre. La distance que les chercheurs québécois ont mise entre leurs recherches et la problématique de la reproduction sociale qui leur était proposée me semble fort révélatrice. Alors que l'ancienneté de la société française prêtait à une analyse des contradictions entre changement et invariance, le contexte historique relativement récent de la société québécoise lui convenait plus mal. À tort ou à raison, ce n'est pas l'invariance qui préoccupe d'abord les chercheurs québécois, mais les changements. Ce qu'ils discutent ici est moins le «processus» de modernisation que le fait lui-même. Il était peut-être un peu tôt, alors que les débats étaient encore tout chauds, pour leur proposer de considérer froidement les permanences d'une société dont on a trop longtemps nié l'entrée dans la modernité. 